\title{
Régulation post-transcriptionnelle de la phosphatase alcaline hépatique
}

L'observation d'une élévation de l'activité des phosphatases alcalines du sérum au cours des maladies du foie et des obstructions des voies biliaires a été faite pour la première fois en 1930. De nombreux travaux ont été consacrés au mécanisme de cette élévation qui est maintenant presque complètement connu, jusqu'à l'échelon moléculaire.

Lorsqu'il devint clair que les élévations les plus importantes de l'activité des phosphatases alcalines du sérum étaient fréquemment observées au cours des obstructions des voies biliaires, les chercheurs proposèrent l'hypothèse selon laquelle cette élévation était due à la "régurgitation" dans le sang de l'enzyme contenue dans la bile. Si cette hypothèse était exacte, l'activité trouvée dans le sérum aurait dî refléter celle de la bile et comprendre les trois isoenzymes : hépatique, intestinale et osseuse. Or les techniques de séparation des isoenzymes appliquées dans les années soixante montrèrent que l'activité sérique était due presque exclusivement à l'isoenzyme hépatique. Cela était incompatible avec la théorie de la régurgitation. L'utilisation du foie perfusé isolé de rat permit alors de montrer que, quelques heures après une ligature $d u$ cholédoque, l'activité de l'enzyme dans le foie était considérablement augmentée et que cette augmentation précédait celle de l'activité sérique. L'élévation de l'activité hépatique était complètement prévenue par les inhibiteurs de la synthèse protéique et de la synthèse de l'ARN. Il devint alors rapidement apparent que l'augmentation de l'activité hépatique et sérique était due à une augmentation de la synthèse de l'enzyme hépatique. Un article de Seetharam et al. [1] tente de préciser le mécanisme moléculaire de l'augmentation de la synthèse. Ces auteurs démontrent que, chez le rat ayant une ligature de la voie biliaire principale, l'augmentation de la synthèse de l'enzyme est due à une augmentation de traduction de l'ARN messager plutôt qu'à une augmentation de la transcription. Les auteurs ont isolé $\mathrm{m} / \mathrm{s} n^{\circ} 10$ vol. 2, décembre 86
l'ARN messager de rats ayant une ligature de la voie biliaire, et de rats témoins. Ils ont. ensuite estimé la quantité d'ARN messager correspondant à la phosphastase alcaline en mesurant ses produits de traduction in vitro au moyen d'un antisérum monospécifique anti-phosphatase alcaline placentaire de rat (qui n'est pas spécifique d'organe). Les résultats indiquent, sans ambiguïté, que la concentration d'ARN messager $n$ 'est élevée ni dans le foie ni dans l'intestin des animaux ayant une ligature du cholédoque, malgré une augmentation de l'activité hépatique $(\times 7)$ et intestinale $(\times 2)$ de l'enzyme. In vivo, l'augmentation de l'activité enzymatique hépatique (et sérique) paraît donc due à une régulation traductionnelle et non transcriptionnelle.

On ne sait pas clairement à l'heure actuelle quel est le signal qui commande cette régulation. Des expériences sur des hépatocytes en culture ont suggéré qu'il pourrait s'agir de l'augmentation de la concentration hépatique des acides biliaires; on ne sait pas non plus par quel mécanisme l'enzyme passe du foie dans le sérum.

En résumé, l'augmentation de l'activité sérique de la phosphatase alcaline est due à une augmentation de sa synthèse hépatique. Cette augmentation de synthèse est régulée chez le rat au stade de la traduction, et non au stade de la transcription. La confirmation définitive de ces conclusions nécessite la production de séquences clonées d'ADN complémentaire. Un ADNc humain codant pour la phosphatase alcaline commune au foie, au rein et à l'intestin vient d'ailleurs d'être isolé [2]

S. E.

1. Seetharam $S$, Sussman NL, Komoda $T$ Alpers DH. The mechanism of elevated alkaline phosphatase activity after bile duct ligation in the rat. Hepatology $1986 ; 6: 374-80$ 2. Weiss MJ, Henthorn PS, Lafferty MA, et al. Isolation and characterization of a cDNA encoding a human liver/bone/kidney-type alkaline phosphatase. Proc Natl Acad Sci USA 1986 83 : 7182-6.
Flash ! c-erb A, équivalent cellulaire de l'oncogène $v$-erb $A$ du virus de l'érythroblastose aviaire, coderait pour le récepteur des hormones thyroïdiennes. Ainsi serait comprise son homologie partielle avec les gènes codant pour les récepteurs des hormones stéroïdes (source: laboratoire de Ron Evans, Salk Institute, San Diego, USA).

\section{BRÈVES}

Il n'existe pas une seule... mais plusieurs protéinekinases $\mathbf{C}$, tel est le résultat le plus important des deux articles récemment publiés dans Science par des chercheurs anglais associés à la firme californienne Genentech. Ces chercheurs ont cloné l'ADNc correspondant à l'enzyme de cerveau de bœuf et l'ont utilisé pour rechercher des séquences partiellement homologues dans des "banques d'ADNc " de différents tissus. Ainsi ont été caractérisés trois types d'ARN messagers codant pour des protéinekinases $\mathrm{C} \alpha$, $\beta$ et $\gamma$. Leurs gènes sont situés sur des chromosomes différents et sont variablement exprimés selon les types cellulaires. Cette multiplicité des protéine-kinases $\mathrm{C}$ pourrait expliquer la diversité des phénomènes physiologiques impliquant cette enzyme.

[Parker PJ et al. Science 1986 ; 233 : 853-9. Coussens L et al. Science 1986 ; 233 : 859-66.]
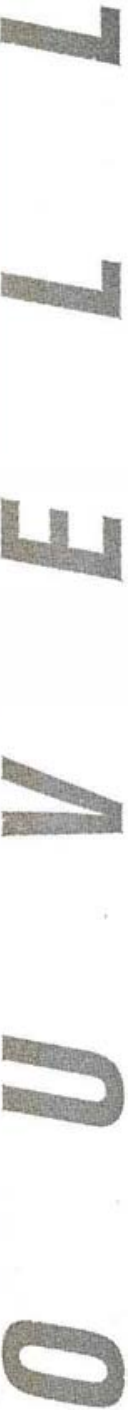

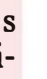

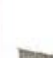

